\title{
Balance Scorecard Application to Predict Business Success with Logistic Regression
}

\author{
Ali Kartal ${ }^{1}$ \\ ${ }^{1}$ Anadolu University, Faculty of Economics and Administrative Sciences \\ Email: akartal@anadolu.edu.tr
}

\begin{abstract}
The purpose of this paper is to show the way of balance scorecard application to develop business failure prediction models. For this reason, first, 43 companies were selected as random sampling among small and medium size carpenters and furniture manufacturer companies in Eskisehir, Turkey. Then collected financial and non-financial data were used in logistic regression that provides us a mathematical equation that has flexibility and easier interpret opportunity.
\end{abstract}

Keywords: Measurements business success; Financial and non-financial indicators; Balance scorecard; and Logistic regression.

\section{$1 \quad$ Introduction}

Business success cannot be explained with using only financial indicators; it should also include nonfinancial indicators. According to this fact, both of these dimensions must be taken simultaneously when we try to estimate the success of business as it was shown on balance scorecard technique by Kaplan and Norton. This approach may also be used to determine the success possibilities of businesses that are in a selected sample group. Then it is possible to separate them as high possibility expected successful company or high possibility expected failure Company. At the same time, it is possible to estimate the degree of how many times a company will be more successful or failure than the others. Under the assumption of balance scorecard application to establish a model and solve it, logistic regression will be used because of its flexibility and easy interview advantages for this kind of studies. To collect data, 43 companies were selected with random sampling method among small and medium size carpenters and furniture manufacturers in Eskisehir, Turkey.

\section{$2 \quad$ Balanced Scorecard Technique and Logistics Regression}

\subsection{Balanced Scorecard Technique}

Although business failure prediction studies are not new, it is the first time that Keasey and Watson advised the use of non-financial symptoms in this kind of prediction models (Keasey \& Watson, 1987). Then Jesus, Quintana, and Gallego showed that non-financial information helps to improve the ability to predict business failure (Jesus, Quintana, and Gallego, 2012). But in their study firm related external variables like national consumer price index, percentage change in legal interest rate was taken as nonfinancial independent variables. Since financial independent variables of the model will include the effect of firm related external variables, under the name of non-financial variables; customer, internal business, and learning and growth dimensions of the company must be taken inside the prediction models. Thus it is necessary to use balanced score card technique in business failure prediction models and we will use it in our study here.

In the contemporary world, to make the right performance measurement, a new structure for performance measurement must be established in that include intellectual assets, motivated and qualified employees, sufficient internal company process, satisficed and royal customers, and so on factors. In this kind of structure, since using of critical financial indicators will be relatively easier than 
using of nonfinancial ones, taking only financial indicators as intensive and nonfinancial indicators for practical use may not be considered as an appropriate method. In other words, managers are dissatisfied with using only financial measures to evaluate business performance because it is not possible to predict future performance expectations by using only past oriented values or results. Moreover, the necessity of connection between business strategy and company's performance measurement this evaluation system should also be taken into account.

To do this, after determining a clear business strategy, taking this strategy into the action, under the light of financial and nonfinancial critical factors, performance measurement system of the company must be developed and prepared accordingly. Under this main idea, three key points given below are very important (Epstein \& Manzoni, 1997)

Companies should establish and use performance measurement systems to support their strategies.

Performance measurement systems should also include nonfinancial indicators.

The company's overall performance measurement system should be broken down into sets of local measurements of lower level units of the company.

Robert KAPLAN and David NORTON formulated this fact as translating strategy into action with four major dimensions as given below diagram.

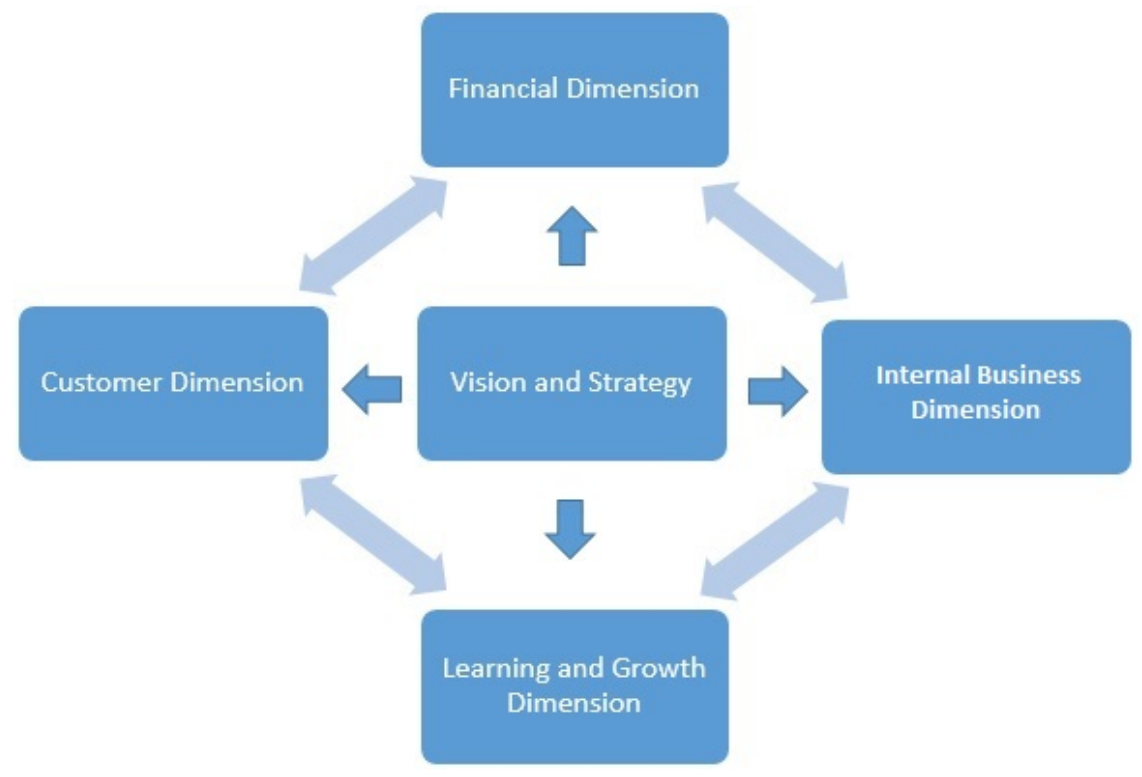

Above given diagram shows the connection between business strategy and performance measurement system of the company and at the same time, it demonstrates both of the critical measurement indicators of the company as financial and nonfinancial indicators. In other words, during the measurement the success of the companies or the prediction company failure studies, above given four dimensions -financial, customer, internal business process, and learning and growth - must be included inside the model and there should be connection with these dimensions and the strategy of the company. After presenting the main discussion about the significance and main structure of the balanced scorecard technique, it is time to explain the necessity of using the logistic regression in this kind of studies.

\subsection{Logistic Regression}

Logistic regression is a regression model in which the dependent variable is categorical like alive vs. dead or profit vs. loss. According to the used scale type and number of the categories of dependent variables, logistic regression can be classified as binary, multinomial or ordinal logistic regression analyses. At the same time, this analysis can also be named as univariate and multivariate logistic regression according to the number of the independent variables. In this analysis, a logistic regression function will be established to estimate the membership of individuals in certain groups with using the relationship between the categorical dependent variable and one or more independent variables. Advantages of using of logistic regression are given below as summary (Çokluk, 2010). 
In this kind of regression model, while the dependent variables are categorical, independent variable or variables may be constant or categorical. This model will not need to meet any requirements of certain possibility distribution assumptions for dependent and independent variables to use it.

Therefore, logistic regression has more flexibility than multiple regression and discriminant analyses and it is easier to establish the mathematical function and interpret the results of the solution. At the same time, in logistic regression, all of the estimated values are positive and they change between 0 and 1. In short, to explain the main differences between multiple regression and logistic regression, it can be said that while the value of dependent variable is being estimated in multiple regression, in logistic regression the realization possibility of the taken values of dependent variable is being estimated. In addition, to do this, while in multiple regression ordinary least square method is used, in logistic regression maximum likelihood method is used. In other words, logistic regression tries to make maximum realization possibility of an event rather than to make minimum the square of variations. As a result, logistic regression provides us a mathematical function that has flexibility and easier interpreting opportunity for constant or categorical independent variables situations.

\section{$3 \quad$ Establishing Model and Interpreting the Results}

To establish the model, among the members of Eskisehir carpenters and furniture manufacturers association 43 companies were selected by using random sampling method and each one was asked four different group questions with face-to-face interview. In the first question; the results of the company for loss or profit, total assets, and total sales were asked to each company. According to the answer on profit or loss situation, all the companies were divided in to two groups as failed and non-failed. This division is taken as dependent variable of our model and these categorical variables were entered to the computer program (SPSS for 15.0) for non-failed as 1 and failed as 0. Then under the light of balance scorecard technique, financial, customer satisfaction, efficiency and learning, and development dimensions were taken as independent variables of the model.

For financial dimension, as an example for financial dimension, total asset turnover that measures the efficiency of the use of capital invested in assets was calculated with using the answer data of the first question. (It is clear that the other financial measurements can also be added in the model in other studies). After calculation, all of the assets turnover rates for our sample companies, with using class number and range that was calculated according to the sturges rule, every company was ranked into the 7 degrees. Here 1 means the lowest and 7 means the highest financial success according to the total assets turnover value of the sample companies.

For customer satisfaction dimension; market share, number of customers, customer satisfaction and so on were examined in the second question. For internal business dimension; service response time, order delivery time, on time delivery, number of main improvements in manufacturing and business process and so on were asked in the third question. For learning and growth dimension; employee satisfaction ratings, percentage of employees trained in process and quality management, percentage of manufacturing process with real time feedback and so on were asked in the fourth question. For the last, three dimensions, under the light of responses to above mentioned item's interviews results, one number was given to each company from 1 to 5 . Here 1 means the lowest value for the dimension of each company and 5 means the highest value of the concerned dimension. Then these numbers were entered into the model with SPSS 15.0). After running the binary logistic regression on the program, the following computer output was taken.

Table 1. Variable in the equation

\begin{tabular}{l|l|l|l|l|l|l}
\hline & B & S.E. & Wald & df & Sig. & $\operatorname{Exp(B)}$ \\
\hline Step Asset turnover & 11.475 & 2258.879 & .000 & 1 & 0.996 & 96320.577 \\
\hline $1 \quad$ Customer satis. & 22.864 & 3218.450 & .000 & 1 & 0.994 & $8.5 \mathrm{E}+009$ \\
\hline Internal business & 10.257 & 4234.941 & .000 & 1 & 0.998 & 28482.008 \\
\hline Learning and growt & 55.576 & 5912.420 & .000 & 1 & 0.993 & $1.4 \mathrm{E}+024$ \\
\hline Constant & -328.365 & 32054.284 & .000 & 1 & 0.992 & .000 \\
\hline
\end{tabular}


In Table 1, B enables us to write the mathematical equation to explain the behaviour of observed groups from the view point of related subject and it gives us constant (fix) value and b values of each independent variables of our model. As it is seen in the table, constant value is $-328,365$ and $b$ values for each independent variable of our model are 11,475, 22,864, 10,257, and 55,576 sequentially. On the other hand, S.E. shows standard errors of constant and b values of independent variables of the model and they give Wald values.

With using above given computer output data, we can write the following equation,

$$
Z=\text { constant }+b 1(X 1)+b 2(X 2)+b 3(X 3)+b 4(X 4)
$$

or

$$
\begin{aligned}
Z= & \text { constant }+b 1(\text { Financial dimension value })+b 2(\text { Customer satisfaction dimension value }) \\
& +b 3(\text { Internal business dimension value })+ \\
& b 4(\text { Learning and development dimension value })
\end{aligned}
$$

and then

$$
\begin{aligned}
Z= & -328,365+11,475(\text { Financial dimension value })+ \\
& 22,864(\text { Customer satisfaction dimension value }) \\
& +10,257(\text { Internal business dimension value })+ \\
& 55,576(\text { Learning and development dimension value })
\end{aligned}
$$

In our study, collected data for the first and second company was like below:

\begin{tabular}{l|l|l}
\hline & First company & Second company \\
\hline Financial dimension & 4 & 1 \\
\hline Customer satisfaction & 4 & 3 \\
\hline Internal business & 5 & 2 \\
\hline Learning \&development & 4 & 2 \\
\hline
\end{tabular}

When reviewing the data of both companies, it is seen that first company has upper grades from all of the independent variables but for the second company financial dimension grade is very weak, customer satisfaction is over the middle, and internal business and learning \& development grades are close to average.

On the created equation (3) with using above given data of each company that was collected from each randomly selected company before, it is possible to calculate $\mathrm{z}$ values of each company. As it is seen below;

$\mathrm{Z}$ value for the first company $=-328,365+11,475(4)+22,864(4)+10,257(5)+55,576(4)$

$\mathrm{Z}$ value for the first company $=82,580$

$\mathrm{Z}$ value for the second company $=-328,365+11,475(1)+22,864(3)+10,257(2)+55,576(2)$

$\mathrm{Z}$ value for the second company $=-116,632$

After these calculated $\mathrm{z}$ values for each company are used in formula $1 /(1+\mathrm{e}-\mathrm{z})$, we will get the potential success probability of each company. If the obtained value for a company is above 0.5, then the particular company is expected to succeed in the future. In the opposite scenario, the company is expected to fail. Then according to these results all of the companies can be separated into two groups as succeed and expected to fail.

For example, for the first company, when the value $\mathrm{z}$ was calculated as 82,580 in the formula $1 /(1+\mathrm{e}-$ $\mathrm{z})$, the result will be close to 1 . Since it is higher than 0.5 , we can expect that the first company will be successful in future. On the other hand, when we make same calculation for the second company, this time result will be close to 0 . Since the result is lower than 0.5 , we can expect that the second company will fail.

This formula enables to determine and separate the companies into two groups, according to their expected success and failure predicted probabilities. Same results can also be seen below as computer output figure 1 . In this figure, 0 shows the companies whose predicted probability is close to 0 or lower than 0.5 and 1 shows the companies whose predicted probability is close to 1 or over 0.50 . It is also 
possible to get these predicted probabilities and predicted groups of each company for the observed group as computer output. As it is seen in figure 1, we had a successful model and result here because all of the observed companies were divided into the two groups as successful and fail expectation and they are seen on the two sides of the predicted probability point 0.50 . If we had some companies around point .50 , it would not be easy to decide the predicted group of them. At the same time, it is possible to calculate that how many times more will be successful a company than other with using odds rates in this process.

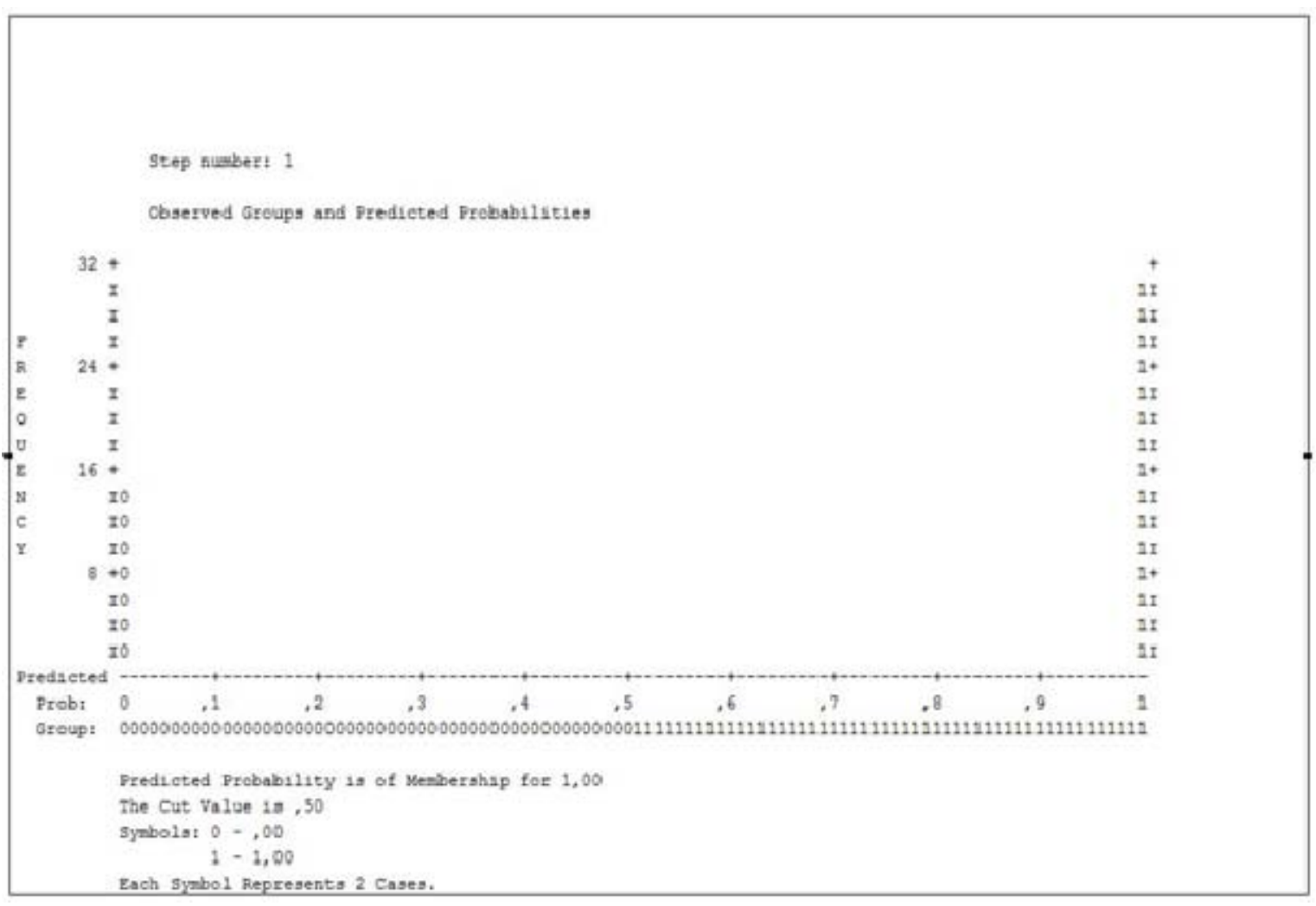

Figure 1. Observed groups and predicted probabilities

\section{Conclusion and Suggestions}

In company success prediction models, financial variables have been used from the past to today. However, we believe that it is not possible to predict tomorrow's performance expectations by using only yesterdays' past oriented numbers or data. Because financial variables of the company are past oriented numbers, therefore, any business failure prediction model should not only consider financial variables but also non-financial variables. And under the name of non-financial variables; customer, internal business, and learning and growth dimensions of the company must be taken inside the prediction models. We included them in our model and showed the way of balance scorecard application to develop business failure prediction models. At the same time, it is necessary to make connection between business strategy and company's performance measurement. For that purpose, balance scorecard application should be used for similar studies and applications.

During this process, we should recognize the advantages of logistic regression that provides us with flexible mathematical function that has easier interpretation opportunity for constant or categorical independent variables situations. In our model, we showed the way of application balanced score card technique to predict business success with logistic regression. As a result, all of the observed companies were divided into the two groups as successful and fail expectation with symbols 0 and 1 in figure 1 . 
Since they are seen on the right and left side of the predicted probability point 0.50 , this is a successful result for us. Because it means that classification table has 100 percent correct situation. This proposed model should be used as more detail in the other studies and applications because it includes not only financial but also non-financial variables.

\section{References}

1. K. Keasey, R. Watson, 'Non-Financial Symptoms and The Prediction of Small Company Failure: A test of Argenti' Hypotheses', Journal of Business Finance and Accounting, 14(3), (Autumn), pp. 335-354, 1987.

2. Maria Jesus, Mures Quintana, and Ana Garcia Gallego, 'On the non-financial information's significance in the business failure models A Spanish case study', International Journal of Organizational Analysis, Vol. 20, No. 4, 2012 .

3. Ö. Çokluk, "Logistics Regression: Concept and Application," Theory \& Practice, vol. 10, no. 3, pp. 1397-407, 2010 .

4. M. J. Epstein and J. F. Manzoni, "Translating Strategy into Action," Management Accounting, vol. 28 (August), 1997.

5. C. T. Horngren, S. M. Datar and M. V. Rajan, Cost Accounting: A Managerial Emphasis Pearson Education Limited, 2012.

6. R. Kaplan and D. Norton, "Using the Balanced Scorecard as a Strategic Management System," Harvard Business Review, January - February, pp. 71-79, 1996 